ELSEVIER

\title{
Neural representation of behavioral outcomes in the orbitofrontal cortex
} Zachary F Mainen ${ }^{1}$ and Adam Kepecs ${ }^{2}$

\begin{abstract}
The orbitofrontal cortex (OFC) is important in processing rewards and other behavioral outcomes. Here, we review from a computational perspective recent progress in understanding this complex function. OFC neurons appear to represent abstract outcome values, which may facilitate the comparison of options, as well as concrete outcome attributes, such as flavor or location, which may enable predictive cues to access current outcome values in the face of dynamic modulation by internal state, context and learning. OFC can use reinforcement learning to generate outcome predictions; it can also generate outcome predictions using other mechanisms, including the evaluation of decision confidence or uncertainty. OFC neurons encode not only the mean expected outcome but also the variance, consistent with the idea that OFC uses a probabilistic population code to represent outcomes. We suggest that further attention to the nature of its representations and algorithms will be critical to further elucidating OFC function.

Addresses

${ }^{1}$ Champalimaud Neuroscience Programme, Instituto Gulbenkian de Ciê ncia, Oeiras P-2781-901, Portugal

${ }^{1}$ Cold Spring Harbor Laboratory, 1 Bungtown Road, Cold Spring Harbor, NY 11724, United States
\end{abstract}

Corresponding author: Mainen, Zachary F (zmainen@igc.gulbenkian.pt) and Kepecs, Adam (kepecs@cshl.edu)

\author{
Current Opinion in Neurobiology 2009, 19:1-7 \\ This review comes from a themed issue on \\ Cognitive neuroscience \\ Edited by Michael Platt and Elizabeth Spelke \\ 0959-4388/\$ - see front matter \\ Published by Elsevier Ltd. \\ DOI 10.1016/j.conb.2009.03.010
}

\section{Introduction}

The orbitofrontal cortex (OFG) was initially characterized as an area whose destruction profoundly impacted human personality, but, paradoxically, left no obvious deficits in standard cognitive tests (reviewed in [1]). Yet, through intensifying scrutiny over the last decade the function of the OFC has arisen from obscurity to take a central place in our understanding of learning and decision-making [2,3]. Today, through a remarkable convergence of studies conducted in species ranging from rats to humans, OFC is widely conceived as a place where the 'value' of things is represented in the brain.
While the concept of 'value' may strike a hard-nosed neuroscientist as hopelessly fuzzy, this concept plays a central role in most behavioral theories of decisionmaking. In neuroeconomic theory, assignment of economic value allows qualitatively different goods to be compared in a single 'universal currency' [4]. In animal learning theory, the similar concept of 'incentive value' measures the ability of outcomes to motivate behavior [5,6]. In machine learning theory, 'state values' and 'action values' are the principal targets of learning and action selection; by maximizing these values, agents learn optimal behavior [7]. By offering formal (i.e. quantitative) definitions of value and related concepts, these theoretical frameworks can help one to test and eventually to understand more precisely what the OFC does. That is because formal definitions can yield concrete predictions that are testable using traditional neurophysiological and behavioral measurements without resorting to semantic arguments about abstract terms [8].

While theoretical perspectives are helpful, they also bring on more work. In the light of theory, questions about OFC function become not only more clear but also more detailed and nuanced, opening up and demanding further experimental tests. Moreover, different theoretical frameworks present partially overlapping, sometimes incongruent, views that must eventually be reconciled. Finally, applying theories of behavior to the brain requires one to bridge the gap between the functional level that forms the basis for the theory and the level of neurophysiology. As famously framed by David Marr [9], two key pieces are needed to bridge between behavioral (computational) and neural (implementational) levels: first, understanding the nature of the neural code or representation; second, understanding the processes or algorithms used to create and utilize these representations.

This review will examine recent progress in OFC function in light of economic, psychological and computational theories of value. While we wholeheartedly acknowledge the convergence of many threads evidence, our main goal is to emphasize the ragged edges and emerging complexities. These become apparent especially when asking what exactly a neural representation within OFG might look like, and therefore our primary focus will be on recordings from individual OFC neurons in monkeys and rats, with secondary attention to lesions and neuroimaging studies. We will also review what we 
know about the origin of these representations, and touch upon the issue of how they are used.

\section{Specific and abstract properties of neural representations in OFC}

OFC was identified in monkeys as an area containing neurons that responded to 'rewarding' substances such as palatable foods but whose activity was not tied directly to their physical attributes: responses could be changed dramatically by associative learning and by the current hunger or satiety of the subject (reviewed by [10]). In this sense, OFG responses reflected something 'subjective' about the value of a reward.

A more precise operational definition of 'subjective value' can be phrased in terms of a decision-maker's preferences amongst different options: assuming that choices on average maximize value, one can infer subjective values from choice preferences [11]. This important idea was tested by Padoa-Schioppa and Assad [12], who recorded single neurons in the OFC while monkeys chose between pairs of juices of different volumes and types. Remarkably, they found neurons whose firing rates were correlated with the relative choice preferences of the animal. Since the preference function combined both the volumes and the types of juice, these response functions were not a simple function of the sensory properties. Nor were they dependent on direction or motor output used to indicate a choice. Thus, OFG neurons can be said to encode 'abstract values', as defined by choice preferences [12].

Signals that correlate with an abstract value satisfy an important need from a neuroeconomic perspective: they provide a common currency for comparisons of unlike goods [4]. But from a computational perspective, these signals seem to raise as many questions as they answer. By definition value is a single scalar variable and can be represented in the firing rate of even a single neuron. So what is all the rest of the 'representational space' of OFC being used for? Or, to put it more simply, what are all those neurons doing? Does value play a role like contrast in the visual cortex, that is a parameter that modulates a primary representation? If so, what is the primary representation? If, alternatively, value is the primary variable, playing a role like spatial location in the primary visual cortex or frequency in the auditory cortex, then what are the other parameters that differentiate the functions of different neurons?

Tellingly, a recent lesion study in rats [28] suggests that the representation of the concrete properties of valuable objects is not merely of secondary importance for OFC function. This study used an intricate conditioning paradigm based on behavior driven by second-order rewards things that are not themselves rewards but are associated with reward (e.g. money). In this situation, the OFC was
Figure 1

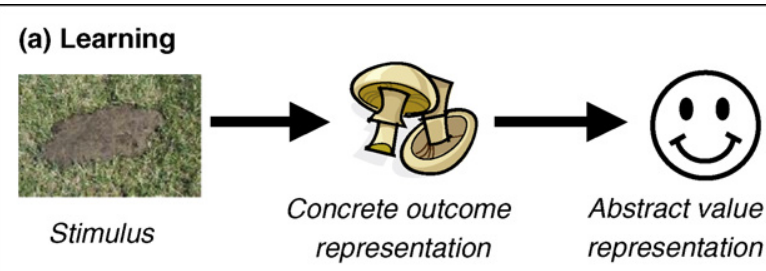

(b) Outcome predictions, normal

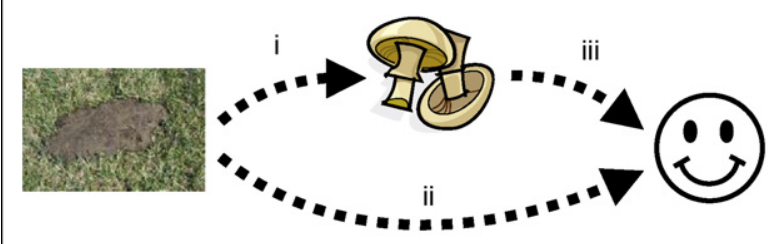

(c) Outcome predictions, OFC lesion

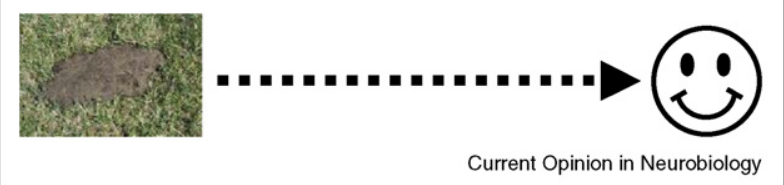

Involvement of OFC in the generation of concrete outcome predictions. Illustration of OFC neural representations underlying second-order conditioning, based on experiments in rats that demonstrate that OFC is required for generating predictions of specific rewarding outcomes but is not required for abstract value predictions [28]. (a) During learning, a neutral stimulus (e.g. cow manure, left) is associated by experience with a rewarding outcome (a type of edible mushroom) which evokes a neural representation of specific properties (appearance, smell, taste and nutrient contents, represented by the drawing in the center) as well as an abstract value (a positive value illustrated by a smiley face, right). (b) In normal intact animals, after learning, exposure to the stimulus (left) generates two types of predictions: those of the specific outcome (center) and those of the abstract or motivational value (right). The dotted arrows (i-iii) illustrate the associative links that underlie these predictions. The specific predictions are generated directly by the stimulus (i). The value predictions are generated both directly by the stimulus (ii) and indirectly through the specific outcome (iii). (c) In animals with OFC lesions, experiments demonstrate that the stimulus still generates abstract value predictions, but fails to generate concrete predictions of the specific outcome, as illustrated. These observations suggest that the key role of OFC is the generation of specific concrete outcome representations.

necessary when predictions of the specific attributes of the reward were utilized but not when only calculating predicted value (Figure 1) However, as we discuss further below, the same OFC neurons can respond both to food items and to the omission of punishments such as electric shocks [31]. More work is needed to define the range of outcome types that might define the 'receptive field' of an OFC neuron.

While deciphering the nature of OFC representations merits further neurophysiological attention, some clues can be gleaned from recent studies. Many attributes of eaten foods, such as fat content, have been found to 
influence activity in OFC (reviewed in [10]). Indeed, in the Padoa-Schioppa and Assad experiments, only one fraction of OFG neurons correlated with abstract preferences; a second class reflected the 'offer value', of only one or two of the juices, while a third class of OFC neurons' firing correlated with physical properties of the juices, being insensitive to the amount $[12,13]$. The selectivity of OFC neurons for specific food properties suggests the possibility that representations in the OFC might be organized according to categories, such as reported in inferior temporal cortex [14] but perhaps based on features such as the caloric and nutrient content of foods.

Another possibility is that OFC representations might be organized using a spatial coordinate system, as found in the visual system, hippocampus, and throughout much of the brain. Indeed, three recent studies indicate that individual OFC neurons recorded in freely moving rats indeed encode spatial locations $[15,16]$ sometimes jointly with value [17]. While OFG responses in monkeys have been reported to be indifferent to the direction of eye movements required to indicate a choice [12], a recent study shows spatial selectivity does arise during the outcome in a task that required monkeys to remember their responses for choices in subsequent trials [58]. It is also important to keep in mind that the brain uses many different kinds of spatial reference frames. If the spatial reference frame in OFC is an allocentric map - "world centered', similar to the one in the hippocampus - then one might not expect to see spatial tuning in head-fixed monkeys with small eye movement. Nonetheless, using suitable manipulations this issue could be also tested in monkeys (cf. [19]).

Finally, since the population of OFG neurons is apparently heterogeneous, do cells with different kinds of selectivity map onto different anatomical substrates? A gradient of abstract to concrete properties from posterior to anterior OFC has been suggested based on neuroimaging [20]. Could more 'abstract' cells that are closer to choice preferences map onto a particular class of cells, for example cortical projection neurons, while other cell types correspond to local neurons? And how are neurons with different functional properties connected as a local network? Are more abstract responses being computed locally by combining more specific ones?

\section{Dynamic updating of values: context, needs and learning}

Central to the concept of values is that they can be dynamically modulated even when the objects of value themselves remain unchanged, and this is a property reflected in OFC. An important example of such dynamic modulation is how the value of a given option depends on the menu of alternatives, called the 'reference frame'. A classic study showed that single neurons in monkey OFC change their response to a given reward depending on the relative value of an alternative reward [21]. This might reflect scaling of OFC representations to fit the available options, allowing neurons with a limited dynamic range of firing rates to represent values over different ranges in different situations.

However, a more recent study using similar methods obtained exactly the opposite result, that the responses of OFG neurons were independent of the alternatives or 'menu' of options [13]. This finding also has a rationale: by keeping a single scale of values one can ensure transitivity of preferences (i.e. if $\mathrm{A}>\mathrm{B}, \mathrm{B}>\mathrm{C}$, then $\mathrm{C}>\mathrm{A}$ ), which is an essential trait for a rational decision maker with consistent choice patterns. Moreover, keeping a single scale avoids the combinatorial explosion of comparisons when many alternatives are present. But how could the discrepancy of these findings be resolved? One possibility is that they are due to differences in methodology. While the first study repeated the same comparison set for large blocks of trials, the second study interleaved different comparison sets from trial-to-trial. Therefore, the contradiction could be resolved by supposing that reference frames change only on time scales longer than a few trials $[13]_{\wedge}$

However, a recent neuroimaging study showed menusensitivity of the activation of human medial OFC using an interleaved trial design [22], apparently contradicting this explanation. This might suggest a second possible explanation, that there is differentiation of function within OFC, with some areas encoding a relatively local reference frame and others a more global one. In any case, it is worth considering that we have not yet gotten a good handle on how subjects apply reference frames or perhaps even how to frame the issue of reference frames $[23,24]$.

A second, important way that values change is depending on the internal state of the organism. Indeed, the OFC appears to play a critical role in the modulation of valuations by internal state. For example, hunger state modulates the subjective value of food and also the firing rates of OFC neurons [25]. This finding implies that the OFC must combine information about the state of the organism's needs with sensory information about the physical attributes of available resources. How this occurs is a critical question that deserves more attention. It is paramount that there is specificity of matching between representations of needs and goods. For instance, hunger signals should enhance the value of food representations while thirst signals should enhance the value of water representations. Such specificity of state-dependent modulation would imply the existence of an attributespecific representational structure in OFC preceding the computation of a purely abstract one. In fact, by recording neuronal populations across feeding cycles in rats, de Araujo and colleagues showed that the ensemble activity 
of OFC neurons can predict satiety-states [26]. It will be fruitful to investigate to what degree of detail an organism's needs are represented in OFC and how representations of needs combined with representations of goods.

The nature of the representational structure may influence how needs combine with rewards in other ways. For instance, a spatial representation [15-17] could allow dynamic updating of outcome values based on their changing relative distance to the agent. In this way, when deciding between two potential food sources, an animal would be able to take distance into account when considering a very abundant food source that is far away from its current location [27]. Such 'spatial discounting' would be very much analogous to temporal discounting demonstrated in OFC neurons (see below).

\section{What are the components of value?}

Value has a number of components and it is somewhat controversial at this moment which ones are represented together or separately in the OFG. First, values have a positive and negative component: $\Lambda$ value $=$ benefit - cost. Human neuroimaging studies tend to indicate that rewards and losses/punishments are processed in distinct subregions of OFC, with lateral regions being more modulated by costs and medial regions by benefits $[20,28,29]$. However, the same neurons that respond to rewards can also signal aversive electrical shocks [30].

A recent neuroimaging study [31] showed that part of OFC correlated with 'willingness to pay', a concept critical in economics that combines cost and benefit.

By contrast, lesion studies in rodents failed to implicate the OFC in processing costs such as the effort of climbing a wall [32,33] or in instrumental behaviors in general [34]. Therefore, it may be important to clarify the differences in calculating and representing costs that reflect negative outcomes, such as receipt of a punishment or a loss, from costs that are associated with the action used to obtain the outcome, such as energy expenditure or transaction costs. Interestingly, a lesion study in monkeys [35] demonstrated that $\mathrm{OFC}$ is required for reinforcement-guided $\Lambda$ decision-making in tasks based on stimulus-outcome associations, but not in tasks that depend on action-outcome associations. Similarly, OFC lesions do not affect valuation during instrumental conditioning in rats [34]. In terms of reinforcement learning, this might reflect a dissociation between a state-value system and an action-value system, with OFC participating in the former but not the latter [36].

It is well-established that OFC activity responds not only to received outcomes, but also to cues that serve to predict such outcomes [37,38] A recent neuroimaging study [39] used a probabilistic task to examine reward value (actual received reward) and expected payoff (average expected reward), finding that both expected value and payoff modulate the same area of OFC. Two components that are essential to valuing predicted outcomes are (1) the probability of occurrence and (2) how far in the future it is expected. A representation of abstract expected value would imply that these distinct components of value (expected time and reward magnitude/probability) are appropriately combined. Interestingly, however, Roesch et al. [16] found single neurons in rat OFC whose activity correlated with both reward delays and sizes independently but not jointly, a result which contrasts with a previous study in monkeys [40]. Expected value signals should also vary inversely with uncertainty. Kepecs et al. [41], by manipulating decision difficulty in a deterministically rewarded categorization task, found that rat OFC neurons can predict outcome probability before receipt. The authors reported two classes of OFC neurons, one whose firing increased with uncertainty and one whose value decreased; the results are discussed in more detail below. In a task where amount, cost and probability were manipulated independently in a single experiment, Kennerley et al. [42] found single neurons in monkey OFG that were modulated by one, two and all three factors, but less commonly than in medial prefrontal cortex.

Although it is evident from these findings that OFC neurons participate in predictive representations of outcomes that incorporate both delays and probabilities, the findings leave much unclear about how these variables are represented within the population of OFC neurons. One interesting possibility that is consistent with the available data, although still speculative, is that predicted outcomes are represented using a probabilistic form of population code, as proposed for other brain areas $[43,44]$. Indeed, there is evidence from neuroimaging that OFC predicts not just expected outcomes (the probability weighted sum of different possible outcomes), but the variance of outcomes as well, sometimes known as 'risk' [33,4547]. Recordings from rat OFC are consistent with a population code for reward value predictions [59]. If OFC neurons are using a probabilistic population code, in which not just the mean estimated outcome, but the full probability distribution of expected outcomes is represented, then both mean and variance of the outcome would be represented simultaneously in the same population of neurons [44].

\section{Computing outcome predictions using reinforcement learning}

We have considered in some detail the properties of neural representations of outcomes or predicted outcomes in OFC. Along with this question of representation comes the question of how these representations, particularly the predictive ones, are generated. In particular, what algorithms can be used to obtain accurate predictions of outcomes? Reinforcement learning (RL) theory provides a normative framework for how to predict and 
obtain maximal values using a two-part procedure: first, learn the values of states (roughly, stimuli) and/or actions; second, select actions in order to maximize predicted future values [7]. The RL framework provides precise normative algorithms for both steps. As parts of these algorithms, there exist abstract variables - including the 'predicted reward value' and 'reward value prediction error' - that can be specified in terms of the history of past stimuli (states), actions and outcomes. By fitting RL models to directly observable behavioral data, one can thus test how well any of these internal variables can predict patterns of behavioral choices or neural activity [48], especially during dynamic situations in which the outcomes themselves or contingencies between stimuli, actions and outcomes vary probabilistically.

Several groups have applied RL model-based approaches to recordings of single neurons in areas including parietal cortex [49,50], striatum [51,52] and prefrontal cortex [53]. What is clear from these studies is that correlates of value and related variables, as defined formally in RL, can be found throughout a distributed network of brain areas, including the OFC. The ubiquity of value signals throughout the brain should not obscure the fact that in most cases these do not appear to represent 'pure value' in a neuroeconomic sense, but rather appear to scale different kinds of sensory or motor representations. However, signals that apparently encode value may also reflect related but computationally distinct variables. Thus, an overarching question is how different variables might be parcellated amongst various brain regions, a task made trickier by the fact that different RL variables are strongly correlated in many tasks. Hare et al. [54], in a recent neuroimaging study, used a task design that allowed them to orthogonalize several related variables. They found that signals in OFC were more closely related to value predictions than to prediction errors. Although model-based approaches have not been applied to single neuron OFG recordings, Takahashi ${ }_{\text {Q1 }}$ et al. (Schoenbaum, in press) used a paradigm with a switch in reward values to test for correlates of value predictions and value prediction errors in OFC. The findings also suggested that OFC encodes value signals but not value error signals. If this is true, then it interesting that OFC neurons encode both the predicted and the actual received outcomes, but do not subtract these signals to produce the prediction error signal that is used for learning, instead leaving this job to dopamine neurons and other areas.

Although reinforcement learning provides an attractive framework for understanding important aspects of OFC function, other aspects remain more challenging. It is known that in environments with complex predictive relationships, OFG neurons are sensitive to these variables [55]. A recent study in monkey OFC demonstrated that OFG neurons do not only signal anticipate rewards across trials, but also maintain a representation of past trial events, with the activity of individual neurons actively retaining information about rewards from one trial to the next [56]. Whereas associative learning mechanisms might act within OFC to generate predictive representations, other forms of outcome prediction appear to require the OFC to work closely with other brain regions. For example, the ability of OFC neurons to predict successive elements of a sequence depends on an intact hippocampus [57]

\section{Computing outcome predictions using confidence estimates}

The outcome value predictions considered in the reinforcement-learning framework above are generated by learning from experience. In principle, outcome pre-

\section{Figure 2}

(a)

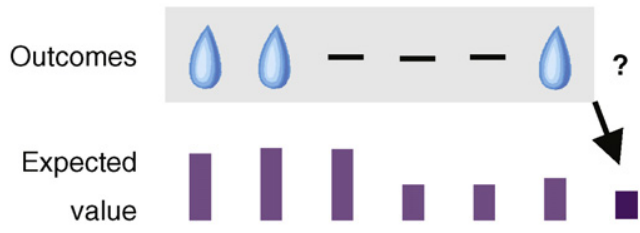

(b)

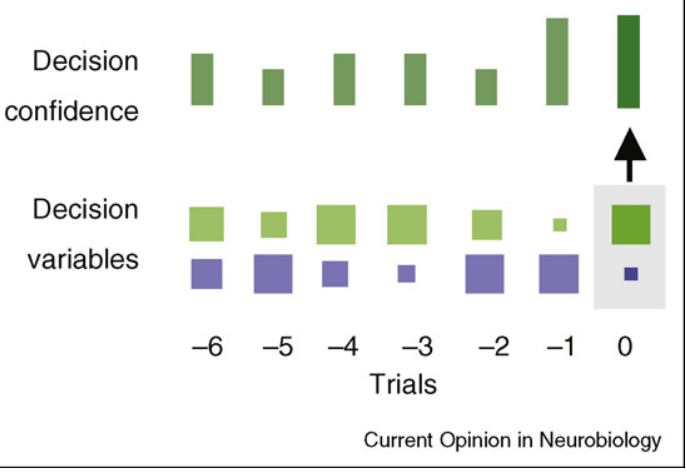

Multiple mechanisms for outcome predictions. Illustration of two mechanisms for generating outcome predictions. (a) In reinforcement learning, the history of past trial outcomes (gray horizontal shading) can be used to predict (arrow) the expected outcome of the current trial (question mark). The expected value on each trial is a weighted sum of previous outcomes (red bars). This prediction mechanism is useful when there is a probabilistic predictive relationship between previous and current outcomes. In such situations the appropriate use of past history can average out stochasticity and provide good outcome predictions. (b) In decision tasks, outcomes can be probabilistic because of limitations or noise in sensory, memory or decision processes. In such situations the most important source of information about the expected outcome is the data on which the decision is based. Therefore, a measurement of the uncertainty of the decision variables (blue and green squares, with size indicating quantity) on the current trial (gray vertical box) can yield a decision confidence estimate that predicts (arrow) the probability of a correct decision (blue-green bars). The relevant decision variables will be different for each kind of decision process, such as sensory and memory variables need for a categorization process. If a correct decision implies reward, such a decision confidence estimate will be a useful predictor of expected reward value as well. For details, see Ref. [42]. 
dictions can be generated by other mechanisms. In many situations, a behavioral outcome depends on a decision that is subject to uncertainty arising from subjective limitations, such as imperfect perception or memory. In such a case, if the decision-maker can assess the quality of the internal representation on which a particular decision is based, this assessment can provide predictive information above and beyond what could be gleaned from past experience.

This process, known as confidence estimation, was examined by Kepecs et al. [41] using a categorization task in which outcomes were deterministic, but decision difficulty could be manipulated by varying the distance of stimuli to the category boundary. The authors showed that using both a standard signal detection theory and an evidence integration-based decision model, it was simple to compute a measure of decision confidence that provided a good estimate of the expected outcome using only information available in the current trial (Figure 2). Remarkably, during outcome anticipation the firing of one third of rat OFC neurons showed the selectivity predicted by such models, a pattern that could not be explained by predictions based on learning from past trial outcomes.

These data suggest that OFC generates outcome predictions not only through reinforcement learning but also by directly accessing internal or subjective information generated during the decision process [41]. By using information derived from internal representations, confidence estimation provides additional means to predict outcomes that is not available through externally observable stimulus-outcome associations. These observations are consistent with the general view that OFG representations concern outcome expectations, but establish a novel means for generating these expectations. It remains to be determined how confidence signals relate to other aspects of OFC representations. In particular, it will be important to determine whether the same neurons that are modulated by confidence are also modulated by other facets of expected value.

\section{Conclusions}

In this review we have emphasized recent progress and open questions in the function of the OFG from a computational perspective. Much evidence points to OFC as representing the ends or outcomes that motivate goal-directed behavior but much remains to be done to flesh out how these highly abstract entities are represented and computed at the level of individual neurons. We suggest that thinking more about how OFC represents information and the algorithms with which it generates and manipulates these representations will lead to more precise design and interpretation of experiments and ultimately a better understanding of how OFC performs its extremely interesting job.

\section{Acknowledgements}

Supported by Champalimaud Foundation (ZFM) and the Whitehall Foundation (AK). We wish to thank $G$ Schoenbaum, J Paton and members of our laboratories for helpful comments on the manuscript.

\section{Uncited reference} [18].

\section{References and recommended reading}

Papers of particular interest, published within the period of review, have been highlighted as:

- of special interest

•• of outstanding interest

1. Dolan RJ: Keynote address: revaluing the orbital prefrontal cortex. Ann N Y Acad Sci 2007, 1121:1-9.

2. Murray EA, O'Doherty JP, Schoenbaum G: What we know and do not know about the functions of the orbitofrontal cortex after 20 years of cross-species studies. J Neurosci 2007 , 27(31):8166-8169

3. Schoenbaum G, Saddoris MP, Stalnaker TA: Reconciling the roles of orbitofrontal cortex in reversal learning and the encoding of outcome expectancies. Ann N Y Acad Sci 2007, 1121:320-335.

4. Montague PR, Berns GS: Neural economics and the biological substrates of valuation. Neuron 2002 , 36(2):265-284

5. Balleine BW, Dickinson A: The role of incentive learning in instrumental outcome revaluation by sensory-specific satiety. Anim Learn Behav 1998, 26:46-59.

6. Rescorla RA: Instrumental responses become associated with reinforcers that differ in one feature. Anim Learn Behav 1990, 18:206-211.

7. Sutton RS, Barto AG: Reinforcement Learning an Introduction, in Adaptive Computation and Machine Learning. Cambridge, Mass. MIT Press; 1998:. (p. xviii, 322 p.).

8. Sugrue LP, Corrado GS, Newsome WT: Choosing the greater of two goods: neural currencies for valuation and decision making. Nat Rev Neurosci 2005, 6(5):363-375.

9. Marr D: Vision: A Computational Investigation into the Human Representation and Processing of Visual Information San Francisco: W.H. Freeman; 1982:. (xvii, 397 p.).

10. Rolls ET, Grabenhorst F: The orbitofrontal cortex and beyond: from affect to decision-making. Prog Neurobiol 2008, 86(3):216-244

11. Tversky A, Kahneman D: The framing of decisions and the psychology of choice. Science 1981, 211(4481):453-458.

12. Padoa-Schioppa C, Assad JA: Neurons in the orbitofrontal cortex encode economic value. Nature 2006, 441(7090):223-226.

13. Padoa-Schioppa C, Assad JA: The representation of economic value in the orbitofrontal cortex is invariant for changes of menu. Nat Neurosci 2008, 11(1):95-102.

14. Kiani Ret al.: Object category structure in response patterns of neuronal population in monkey inferior temporal cortex. J Neurophysiol 2007, 97(6):4296-4309.

15. Feierstein CE et al.: Representation of spatial goals in rat orbitofrontal cortex. Neuron 2006, 51(4):495-507.

16. Roesch MR, Taylor AR, Schoenbaum G: Encoding of timediscounted rewards in orbitofrontal cortex is independent of value representation. Neuron 2006, 51(4):509-520. 
17. Furuyashiki T, Holland PC, Gallagher M: Rat orbitofrontal cortex separately encodes response and outcome information during performance of goal-directed behavior. J Neurosci 2008, 28(19):5127-5138.

18. Deaner RO, Platt ML: Reflexive social attention in monkeys and humans. Curr Biol 2003, 13(18):1609-1613.

19. Dean HL, Platt ML: Allocentric spatial referencing of neuronal activity in macaque posterior cingulate cortex. $J$ Neurosci 2006, 26(4):1117-1127.

20. Kringelbach ML, Rolls ET: The functional neuroanatomy of the human orbitofrontal cortex: evidence from neuroimaging and neuropsychology. Prog Neurobiol 2004, 72(5):341-372.

21. Tremblay L, Schultz W: Relative reward preference in primate orbitofrontal cortex. Nature 1999, 398(6729):704-708.

22. Elliott R, Agnew Z, Deakin JF: Medial orbitofrontal cortex codes relative rather than absolute value of financial rewards in humans. Eur J Neurosci 2008, 27(9):2213-2218.

Q4 23. Hsee CK et al.: Preference reversals between joint and separate evaluations of options: a review and theoretical analysis. Psychol Bull 1999, 125:576-590.

24. Seymour B, McClure SM: Anchors, scales and the relative coding of value in the brain. Curr Opin Neurobiol 2008, 18(2):173-178.

25. Critchley HD, Rolls ET: Hunger and satiety modify the responses of olfactory and visual neurons in the primate orbitofrontal cortex. J Neurophysiol 1996, 75(4):1673-1686.

26. de Araujo IE et al:: Neural ensemble coding of satiety states. Neuron 2006, 51(4):483-494.

27. Stevens JR et al.: Will travel for food: spatial discounting in two new world monkeys. Curr Biol 2005, 15(20):1855-1860.

28. Liu $\mathrm{X}$ et al.: Functional dissociation in frontal and striatal areas for processing of positive and negative reward information. $J$ Neurosci 2007, 27(17):4587-4597.

29. O'Doherty $\mathrm{J}$ et al.: Abstract reward and punishment representations in the human orbitofrontal cortex. Nat Neurosci 2001, 4(1):95-102.

30. Hosokawa Tet al.: Neurons in the macaque orbitofrontal cortex code relative preference of both rewarding and aversive outcomes. Neurosci Res 2007, 57(3):434-445.

31. Plassmann H, O'Doherty J, Rangel A: Orbitofrontal cortex encodes willingness to pay in everyday economic transactions. J Neurosci 2007, 27(37):9984-9988.

32. Rudebeck $\mathrm{PH}$ et al.: Separate neural pathways process different decision costs. Nat Neurosci 2006, 9(9):1161-1168.

33. Rushworth MF, Behrens TE: Choice, uncertainty and value in prefrontal and cingulate cortex. Nat Neurosci 2008, 11(4):389-397.

34. Ostlund SB, Balleine BW: Orbitofrontal cortex mediates outcome encoding in Pavlovian but not instrumental conditioning. J Neurosci 2007, 27(18):4819-4825.

35. Rudebeck PH et al.: Frontal cortex subregions play distinct roles in choices between actions and stimuli. J Neurosci 2008 28(51):13775-13785.

36. Balleine BW, Daw N, O'Doherty J: Multiple forms of value learning and the function of dopamine. In Neuroeconomics: Decision Making and the Brain. Edited by Glimcher P et al.: Academic Press; 2008:365-388.

37. Schoenbaum G, Roesch M: Orbitofrontal cortex, associative learning, and expectancies. Neuron 2005, 47(5):633-636.

38. Wallis JD: Orbitofrontal cortex and its contribution to decisionmaking. Annu Rev Neurosci 2007, 30:31-56.
39. Rolls ET, McCabe C, Redoute J: Expected value, reward outcome, and temporal difference error representations in a probabilistic decision task. Cereb Cortex 2008, 18(3):652-663.

40. Roesch MR, Olson CR: Neuronal activity in primate orbitofrontal cortex reflects the value of time. $J$ Neurophysiol 2005, 94(4):2457-2471.

41. Kepecs A et al:: Neural correlates, computation and behavioural impact of decision confidence. Nature 2008, 455(7210):227-231.

42. Kennerley SW et al.: Neurons in the frontal lobe encode the value of multiple decision variables. $J$ Cogn Neurosci 2008.

43. Pouget $A$, Dayan $P$, Zemel R: Information processing with population codes. Nat Rev Neurosci 2000, 1(2):125-132.

44. Beck JM et al.: Probabilistic population codes for Bayesian decision making. Neuron 2008, 60(6):1142-1152.

45. Hsu $\mathrm{M}$ et al.: Neural systems responding to degrees of uncertainty in human decision-making. Science 2005, 310(5754):1680-1683.

46. Tobler PN et al:: Reward value coding distinct from risk attitude-related uncertainty coding in human reward systems. Journal of neurophysiology 2007, 97(2):1621-1632.

47. Pais-Vieira M, Lima D, Galhardo V: Orbitofrontal cortex lesions disrupt risk assessment in a novel serial decision-making task for rats. Neuroscience 2007, 145(1):225-231.

48. Corrado G, Doya K: Understanding neural coding through the model-based analysis of decision making. J Neurosci 2007, 27(31):8178-8180.

49. Sugrue LP, Corrado GS, Newsome WT: Matching behavior and the representation of value in the parietal cortex. Science 2004 304(5678):1782-1787.

50. Dorris MC, Glimcher PW: Activity in posterior parietal cortex is correlated with the relative subjective desirability of action. Neuron 2004, 44(2):365-378.

51. Samejima K et al.: Representation of action-specific reward values in the striatum. Science 2005, 310(5752):1337-1340.

52. Lau B, Glimcher PW: Value representations in the primate striatum during matching behavior. Neuron 2008, 58(3):451463.

53. Barraclough DJ, Conroy ML, Lee D: Prefrontal cortex and decision making in a mixed-strategy game. Nat Neurosci 2004 7(4):404-410.

54. Hare TA et al.: Dissociating the role of the orbitofrontal cortex and the striatum in the computation of goal values and prediction errors. J Neurosci 2008, 28(22):5623-5630.

55. Schoenbaum G, Eichenbaum H: Information coding in the rodent prefrontal cortex. I. Single-neuron activity in orbitofrontal cortex compared with that in pyriform cortex. J Neurophysiol 1995, 74(2):733-750.

56. Simmons JM, Richmond BJ: Dynamic changes in representations of preceding and upcoming reward in monkey orbitofrontal cortex. Cereb Cortex 2008, 18(1):93-103.

57. Ramus SJ et al.: Interactions between the orbitofrontal cortex and the hippocampal memory system during the storage of long-term memory. Ann N Y Acad Sci 2007, 1121:216-231.

58. Tsujimoto S, Genovesio A, Wise SP: Monkey orbitofrontal cortex encodes response choices near feedback time. J Neurosci 2009, 29(8):2569-2574.

59. van Duuren E, Lankelma J, Pennartz CM: Population coding of reward magnitude in the orbitofrontal cortex of the rat. $J$ Neurosci 2008, 28(34):8590-8603. 